\title{
Percepção de gestores e enfermeiros sobre a organização do fluxo assistencial na rede de serviços de saúde
}

\section{Perception of managers and nurses about the organization of care flow in the health services network}

\section{Percepción de gestores y enfermeros sobre la organización del flujo asistencial en la red de servicios de salud}

Kamilla Santos Silva | kamillas.enfufba@gmail.com

Universidade Federal da Bahia. Campus Anísio Teixeira. Instituto Multidisciplinar em Saúde. Vitória da Conquista, Brasil.

Adriano Maia dos Santos |maiaufba@ufba.br

Universidade Federal da Bahia. Campus Anísio Teixeira. Instituto Multidisciplinar em Saúde. Vitória da Conquista, Brasil.

Jamille Amorim Carvalho |jmilleamorim@gmail.com

Secretaria Estadual de Saúde da Bahia (SESAB). Salvador, Brasil.

Clávdia Nicolaevna Kochergin |clavdian@yahoo.com.br

Universidade Federal da Bahia. Campus Anísio Teixeira. Instituto Multidisciplinar em Saúde. Vitória da Conquista, Brasil.

Patty Fidelis de Almeida | patty.fidelis@hotmail.com

Universidade Federal Fluminense (UFF). Departamento de Planejamento em Saúde. Instituto de Saúde Coletiva. Niterói, Brasil.

\section{Resumo}

Este artigo analisa como gestores e trabalhadores da saúde organizam o fluxo assistencial dos usuários quando há necessidade de compartilhamento de cuidados entre diferentes níveis de atenção à saúde. Tratase de pesquisa qualitativa com base em entrevistas semiestruturadas com cinco gestores e seis enfermeiros da Estratégia Saúde da Família (ESF) em um município da Bahia. Os resultados indicam redução na busca direta de hospitais, fortalecimento da Estratégia Saúde da Família como porta de entrada principal para os diagnósticos e participação fundamental do agente comunitário de saúde na organização dos fluxos assistenciais. Porém, observa-se insuficiente informatização das unidades de saúde e determinação de prazos para serviços especializados, realizada pela central de regulação de procedimentos especializados, de forma burocrática, sem coordenação da equipe de atenção primária à saúde. A organização do fluxo assistencial é dificultada pela incipiência da rede de atenção à saúde e ausência de instrumentos de comunicação interprofissional.

Palavras-chave: atenção primária à saúde; acesso aos serviços de saúde; profissional de saúde; atenção secundária à saúde. 


\begin{abstract}
This article analyzes how health managers and workers organize the care flow of users when there is a need to share care between different levels of health care. This is a qualitative research based on semi-structured interviews with five managers and six nurses of the ESF - Estratégia Saúde da Família (Family Health Strategy) in a municipality of the state of Bahia. The results indicate a reduction in the direct search for hospitals, the strengthening of the ESF as the main entry to diagnoses and the fundamental participation of the community health worker in the organization of care flows. However, there is insufficient computerization of the health units and bureaucratic determination of deadlines for specialized services, carried out by the specialized procedures regulation central, without coordination of the primary health care team. The organization of care flow is hampered by the incipience of the health care network and by absence of inter professional communication tools.
\end{abstract}

Keywords: primary health care; health services accessibility; health worker; secondary health care.

\title{
Resumen
}

Este artículo analiza como los gestores y trabajadores de la salud organizan el flujo asistencial de los usuarios cuando existe la necesidad de compartir cuidados entre diferentes niveles de atención a la salud. Se trata de una investigación cualitativa basada en entrevistas semiestructuradas con cinco gestores y seis enfermeros de la ESF - Estratégia Saúde da Família (Estrategia de Salud de la Familia) en un municipio del estado de Bahía. Los resultados indican una reducción de la búsqueda directa de hospitales, un fortalecimiento de la ESF como puerta de entrada principal para los diagnósticos y la participación fundamental del agente comunitario de salud en la organización de los flujos asistenciales. Sin embargo, la informatización de las unidades de salud es incipiente y la central de consultas de los procedimientos especializados programa los servicios de forma burocrática, sin la coordinación del equipo de atención primaria de salud. La organización del flujo asistencial se dificulta por el uso de medidas incipientes en la red de servicios de salud y por la ausencia de instrumentos de comunicación interprofesional.

Palabras clave: atención primaria de salud; accesibilidad a los servicios de salud; personal de salud; atención secundaria de salud.

\begin{abstract}
Contribuição dos autores: Kamilla Santos Silva coletou, organizou e analisou os dados. Escreveu o artigo e aprovou a versão final. Adriano Maia dos Santos orientou a pesquisa. Revisou o texto e aprovou a verão final. Jamille Amorim Carvalho analisou os dados, revisou o texto e aprovou a versão final. Clávdia Nicolaevna Kochergin analisou os dados, revisou o texto e aprovou a versão final. Patty Fidelis de Almeida analisou os dados, revisou o texto e aprovou a versão final.
\end{abstract}

Declaração de conflito de interesses: não houve.

Fontes de financiamento: $\mathrm{CNPQ}$.

Considerações éticas: a pesquisa foi aprovada pelo Comitê de Ética em Pesquisa, da Universidade Federal da Bahia (parecer $n^{0}$ 573.837), em 31/03/2014.

Histórico do artigo: Submetido: 24.dez.2016 | Aceito: 08.maio.2017 | Publicado: 30.jun.2017

Licença CC BY-NC atribuição não comercial. Com essa licença é permitido acessar, baixar (download), copiar, imprimir, compartilhar, reutilizar e distribuir os artigos, desde que para uso não comercial e com a citação da fonte, conferindo os devidos créditos de autoria e menção à Reciis. Nesses casos, nenhuma permissão é necessária por parte dos autores ou dos editores. 


\section{Introdução}

Dificuldades de acesso aos serviços de média densidade tecnológica são um dos entraves para consecução da integralidade assistencial no Sistema Único de Saúde (SUS) ${ }^{1}$. Os gestores deparam-se por um lado com a tripla carga de doença ${ }^{2}$, simultaneamente ao desenvolvimento de novas técnicas e equipamentos de diagnóstico e terapia que se somam aos existentes, além da pressão de diversos grupos de interesse para incorporação e disponibilidade nos serviços de saúde ${ }^{3}$, reforçando a necessidade de mecanismos públicos de regulação, na perspectiva de assegurar o acesso com equidade.

Por outro lado, a maioria dos municípios não consegue ofertá-los de forma integral, dentro do seu território, de modo a responder adequadamente às diferentes necessidades sanitárias ${ }^{4}$. A fragmentação dos sistemas de saúde, caracterizada por um conjunto de pontos de atenção isolados e desarticulados que, por consequência, são incapazes de prestar uma atenção contínua à população, pode ser superada a partir de serviços de saúde organizados em redes de atenção regionalizadas ${ }^{2,4}$. Neste contexto, é fundamental que haja a coordenação assistencial, que consiste em um "atributo organizacional dos serviços de saúde que se traduz na percepção de continuidade dos cuidados na perspectiva do usuário”.

Pesquisas evidenciam alcance de maior integração entre níveis assistenciais e melhor coordenação das condições crônicas em países com atenção primária à saúde (APS) forte ${ }^{6}$; além de redução das hospitalizações desnecessárias e melhor nível de saúde das pessoas, devido à coordenação do cuidado e maior abrangência da APS7. Para além das inovações que podem contribuir para melhor coordenação, sistemas de saúde com forte base de APS apresentam maiores possibilidades de enfrentamento da fragmentação do cuidado ${ }^{8}$.

A coordenação constitui o vínculo entre profissionais da APS e os profissionais dos demais pontos da rede, de forma a organizar as diversas ações e serviços essenciais de acordo com as necessidades dos usuários, permitindo fluxos de cuidado continuado ${ }^{9}$. No Brasil, um amplo estudo que utilizou informações do Programa Nacional de Melhoria do Acesso e da Qualidade da Atenção Básica (PMAQ-AB) considerou que persistem importantes barreiras organizacionais para acesso, que os fluxos assistenciais estão pouco ordenados, a integração da APS à rede é incipiente e há fragilidade na coordenação entre APS e atenção especializada ${ }^{10}$.

Portanto, não basta ampliação da Estratégia Saúde da Família (ESF), ou seja, não é suficiente a transposição do lócus de trabalho do ambulatório para o domicílio ou do hospital para a unidade de saúde da família (USF), quando os processos de trabalho permanecem inalterados, ou mesmo quando imprimem uma nova lógica de produção, mas o núcleo tecnológico conserva-se centrado no procedimento/doença e não no usuário ${ }^{11}$. Essa é uma das razões para a ESF permanecer como produtora de demandas e pouco resolutiva ${ }^{11}$.

Este artigo analisa como gestores e trabalhadores da saúde, no município de Livramento de Nossa Senhora, Bahia, organizam o fluxo assistencial dos usuários quando há necessidade de compartilhamento de cuidados entre diferentes níveis de atenção à saúde. Compreende-se que a organização do fluxo assistencial é uma das dimensões essenciais para a consecução de cuidados coordenados, fazendo parte do conjunto de estratégias necessárias à integração das redes de atenção à saúde ${ }^{5,12}$.

\section{Metodologia}

Trata-se de estudo de caso com abordagem qualitativa ${ }^{13}$, realizado no município de Livramento de Nossa Senhora, na região de saúde de Brumado, sudoeste da Bahia, distante $586 \mathrm{~km}$ da capital do estado. Foram realizadas 11 entrevistas semiestruturadas, com cinco profissionais da gestão municipal e seis enfermeiros da ESF, além de observações em USF, entre abril e maio de 2014. Levou-se em consideração o critério de saturação para definição do número de participantes ${ }^{14}$.

Resultados e discussão são apresentados em dois eixos que traduzem as categorias utilizadas para analisar o processo de organização dos fluxos assistenciais: 1) Desafios à coordenação do cuidado nas 
práticas assistenciais das equipes; 2) Dispositivos informacionais utilizados para organização do fluxo assistencial na rede de atenção. Optou-se pela análise de conteúdo temática ${ }^{13}$, constituída pela ordenação e classificação dos dados, sistematizando-os em núcleos de sentido e leitura transversal, priorizando recortes temáticos mais relevantes relacionados ao objeto de estudo e, por fim, análise dos resultados por meio do entrecruzamento das informações das distintas fontes.

\section{Resultados e discussão}

\section{Desafios à coordenação do cuidado nas práticas assistenciais das equipes}

No município de Livramento de Nossa Senhora, a Estratégia Saúde da Família encontrava-se em consolidação como porta de entrada preferencial para os usuários na rede local, apesar de coexistir o acesso inicial por meio de outros estabelecimentos de saúde. Nesse sentido, os enfermeiros revelaram situações de redução da busca direta de serviços hospitalares, sobretudo para casos que podiam ser resolvidos no âmbito da ESF. Contudo, os mesmos profissionais admitiram que paralelamente à consolidação da unidade de saúde da família (USF) como lócus principal para os primeiros cuidados, convivia-se, ainda, com a busca direta pelos usuários da central de regulação e marcação de consultas, muitas vezes, sem prévia avaliação clínica dos profissionais das equipes de saúde da família (EqSF).

Tais constatações são sintetizados nos depoimentos a seguir:

Acredito que melhorou bastante[ESF como porta de entrada principal], melhorou muito principalmente nos últimos quatro anos. [...] Eles [usuários] deixam mesmo para ir ao hospital só no caso de urgência e emergência. (E1, enfermeiro da ESF)

[...] ainda existem aqueles [usuários] que procuram hospital, procuram a central [de marcação de consultas] por livre e espontânea vontade, até pela demora [...] acham que a gente [EqSF] não está mandando o malote [com solicitação de consultas]. (E6, enfermeiro da ESF)

Compreende-se a EqSF como catalisadora na construção de vínculos com a comunidade, responsável pela resolubilidade no primeiro nível de atenção, além de possibilitar a continuidade e longitudinalidade da atenção $0^{10,11,15}$. Nesse sentido, as EqSF no município realizavam ações visando fortalecer, no usuário, a percepção da USF como porta de entrada preferencial. Assim, entrevistados relataram dispor, como estratégias de orientação, de salas de espera nas USF, visitas domiciliares dos agentes comunitários de saúde (ACS), palestras em escolas e outros espaços comunitários. Além disso, para alguns entrevistados, permanecia uma imagem distorcida do SUS, como serviços voltados apenas para população carente, além de desconfiança por parte da população, quanto à organização dos fluxos pela EqSF.

Assim, visando superar alguns entraves, foi relatado, por enfermeiros, que as EqSF discutiam com a população a organização e o funcionamento do sistema local saúde, abordando como os serviços eram prestados e a forma de acesso aos diferentes níveis de atenção na rede de atenção à saúde. Os fragmentos adiante resumem tais estratégias:

[...] nossa estratégia é mais da visita do ACS, que vai nas casas e orienta que qualquer coisa que eles [usuários] precisarem procurem primeiro a unidade de saúde. Quando eles [usuários] estão aqui [na USF], também, fazendo consulta comigo, têm a sala de espera; a gente, às vezes, orienta que qualquer coisa que precisar deve procurar primeiro a unidade. (E3, enfermeiro da ESF)

[...] salas de espera, palestras na própria unidade, palestras nas escolas, nos espaços em que a própria população tem, conscientizando a população que primeiro é a unidade. (E5, enfermeiro da ESF) 
[...] A gente senta e conversa [com o usuário], pede ao ACS para fazer busca ativa e tal, para conversar, mostrar para eles [usuários] o papel do PSF. (E6, enfermeiro da ESF)

As entrevistas indicam que tais iniciativas vêm colaborando para o processo organizacional da assistência, pois usuários são sensibilizados acerca dos fluxos na rede. Assim, itinerários que permeiam ações de prevenção, cura e reabilitação são esclarecidos aos usuários, permitindo que os mesmos, gradativamente, superem a visão centrada no hospital. Nesta perspectiva, as equipes esperam que os usuários identifiquem a USF como a porta de entrada preferencial do sistema de saúde, seja para lidar com problemas de saúde em diferentes fases da vida, bem como, para o encaminhamento adequado para outros serviços e continuidade do cuidado ao longo do tempo.

Entretanto, além de garantir ações educativas, é essencial que as EqSF compreendam e assumam sua responsabilidade técnica-ética-política na gestão do cuidado ${ }^{16}$. Além da assistência, os profissionais devem extrapolar sua área de saber específica e envolver-se com o cotidiano da comunidade de forma a interagir com os distintos sujeitos do seu território para efetivação de práticas mais abrangentes ${ }^{17}$.

Em relação ao processo de trabalho das EqSF, gestores e profissionais entrevistados reconheceram que tanto médicos quanto enfermeiros realizavam atividades educativas, consultas individuais, conforme sua competência profissional e encaminhamentos para outros serviços (sobretudo, médicos), por meio das fichas de solicitação de exames/consultas que eram direcionadas à central de regulação e marcação de consultas municipal. Contudo, a gerência da USF e as ações administrativas ficavam sob responsabilidade, quase que exclusiva, dos enfermeiros, diminuindo as suas possibilidades de atuar na coordenação do cuidado clínico dos usuários.

Os depoimentos reportam as questões debatidas:

[...] No caso dos médicos, eles vão avaliar pacientes e fazer os encaminhamentos. [...] Elas [enfermeiras] fazem encaminhamentos que cabem à enfermagem; orientam, também, os pacientes para que instituição procurar, como vai ser feita essa marcação, tanto marcação [de consultas] como qualquer outro procedimento. (E9, gestor em saúde)

[...] médicos têm alguns sim comprometidos com a saúde coletiva, mas não são todos. O grande comprometimento mesmo é dos enfermeiros [...]. Nossa revelação tem sido os médicos cubanos, pois são bem comprometidos com a parte de saúde coletiva. (E7, gestor em saúde)

Além dos atendimentos de enfermagem, fazemos os [serviços] burocráticos, [preencher] os papéis que a gente manda [para a Secretaria de Saúde] para alimentar o sistema [de informação]. (E2, enfermeiro da ESF)

Ainda sobre a gerência das USF, observações em campo, bem como as entrevistas evidenciaram o trabalho de destaque da enfermagem. Entretanto, excesso de ações burocráticas no cotidiano das EqSF resultava em sobrecarga de trabalho e dificuldades na execução de ações assistenciais. Neste aspecto, também, experiências internacionais ${ }^{18}$ demonstram que, ao assumir funções clínicas, o enfermeiro da APS pode ofertar atenção em saúde de qualidade similar à prestada pelo médico, além de ser capacitado para exercício de funções de coordenação com gestão clínica e de casos, gerenciando o cuidado na rede.

Nesse sentido, com longas listas de usuários e aumento do número de tarefas a serem desenvolvidas, médicos de APS têm dificuldade de prover atendimentos de alta qualidade e ações de prevenção em curto espaço de tempo, e ainda de desenvolver atividades de coordenação do cuidado para os quais não há esquemas de remuneração ${ }^{8}$. As ações de coordenação provavelmente irão falhar se estiverem sob a responsabilidade exclusivamente de médicos. Enfatiza-se, portanto, a necessidade do fortalecimento do trabalho em equipe e esquemas de pagamento assim como de organização do trabalho que privilegiem não só as consultas, mas, por exemplo, o tempo entre elas para ações de coordenação ${ }^{8}$. 
Nos relatos dos entrevistados, outro sujeito mencionado como importante para coordenação do cuidado foi o ACS. O trabalho do ACS nas EqSF demonstrou-se essencial, inclusive para viabilização da comunicação entre equipe e comunidade, possibilitando que ações desenvolvidas por profissionais de cunho individual (consultas) ou coletivas (educação em saúde) sejam amplamente difundidas entre as pessoas do território de adscrição. Os entrevistados, além de reconhecerem a dimensão comunicacional do ACS, destacaram seu trabalho imprescindível para coordenação do cuidado, no momento em que vão de casa em casa esclarecendo o local de atendimento previamente agendado, entregando resultados de exames e/ou comunicando o agendamento de consultas/procedimentos com especialistas.

Os depoimentos de enfermeiros destacam o protagonismo do ACS:

[...] É através deles [ACS] que a gente consegue concluir nosso trabalho para tudo, na questão de informação, na questão de entrega de marcação de exames, são [ACS] nosso elo nas informações. (E5, enfermeiro da ESF)

Quando há campanha de vacina, além dos meios de comunicação, os ACS passam de casa em casa, a gente faz o levantamento, tudo através dos agentes. (E3, enfermeiro da ESF)

Então a gente [ACS e enfermeiro] é bem articulado, não tem dificuldade nenhuma em relação a isso. $O$ contato com a comunidade é pelo agente comunitário de saúde. (E4; enfermeiro da ESF)

Assim, o ACS é um agenciador de encontros entre territórios existenciais, posto que enlaça saberes populares e conhecimentos técnicos em saúde com potencial para fortalecer os vínculos da população com a EqSF e, assim, ampliar os cuidados ${ }^{19}$. Dessa maneira, na realidade de territórios dispersos, em zonas de difícil acesso às residências e na precariedade dos sistemas comunicacionais, o ACS apresenta-se como elemento essencial para a coordenação do cuidado na realidade do SUS.

A percepção de gestores e profissionais converge para resultados encontrados em estudo realizado em regiões de saúde da Bahia, no qual, o ACS foi o profissional mais envolvido na busca de informações e visitas durante casos de internação, indicando potencialidades deste profissional para maximizar as ações de coordenação empreendidas pela ESF no território ${ }^{12}$.

\section{Dispositivos informacionais utilizados para organização do fluxo assistencial na rede de atenção}

O debate na literatura internacional sustenta a importância da coordenação do cuidado, fortemente apoiada por dispositivos informacionais que facilitem a comunicação entre prestadores ${ }^{6,7,9}$. Assim, para que a APS seja o centro da atenção na rede assistencial, todas as informações devem estar disponíveis para seus profissionais e usuários; ou seja, múltiplos provedores independentes, sem um eixo articulador, não conseguem definir claramente suas responsabilidades ${ }^{8}$.

Para identificação nos serviços, os usuários dispunham, além do cartão SUS, os cartões da família, do Hiperdia, da gestante, do idoso, entre outros. Todavia, um dos entrevistados relatou a resistência de algumas pessoas aos serviços do SUS e a percepção de que não precisam do sistema público e, por isso, o cadastramento seria algo desnecessário, especialmente para aqueles que possuíam algum seguro privado de saúde.

Os relatos, a seguir, destacam tais considerações:

Tem o cartão da família, se o paciente for hipertenso ou se for diabético tem, também, o cartão do hiperdia, temos o cartão SUS [...] nós temos esses cartões, gestante tem cartão, criança, idoso... (E7, gestor em saúde)

[...] alguns [usuários] falam que têm o plano, preferem ir para um centro maior como Vitória da Conquista, eles preferem frequentar clínicas com mais aparelhagem. (E1, enfermeiro da ESF) 
Tem cartão da família, mas nem todos [usuários] estão cadastrados no cartão SUS. [...] alguns ainda têm resistência em tirar, [e eles dizem]: - eu nunca vou precisar do posto, para que fazer esse cartão do SUS? (E3, enfermeiro da ESF)

[...] as pessoas [usuários] que têm condição não pegam remédio do posto. Eles [usuários] falam até assim: - ah, não presta, não é a mesma coisa! (E4, enfermeiro da ESF)

Tais evidências são desafios à coordenação do cuidado, sobretudo pela desvalorização social do SUS, especialmente da APS. Neste sentido, os profissionais da ESF enfrentam, além da precariedade real do sistema de comunicação entre os diferentes pontos da rede, a imagem simbólica de trabalharem num serviço de menor importância ou, ainda, de serem desprestigiados pelos usuários e mesmo pelos pares que atuam em pontos mais adensados tecnologicamente.

Quanto às alternativas de integração das informações clínicas, o município contava com fichas de referência e contrarreferência, com as quais, profissionais, apenas no nível local, podiam compartilhar informações, ainda que de maneira limitada. Em que pese a simplicidade dos dispositivos informacionais mencionados, estes eram relevantes para a organização dos fluxos e contrafluxos de pessoas na rede local. Entretanto, quanto à contrarreferência, muitos especialistas não forneciam essa ficha de retorno, dificultando a integração das informações entre diferentes pontos da rede assistencial, situação, também, encontrada em outros estudos ${ }^{10,12}$.

Tal problema era mais um obstáculo à coordenação adequada entre níveis:

[...] a gente vê que a contrarreferência ainda está muito a desejar, porque a gente faz a referência, mas na verdade a contrarreferência é um nó crítico. (E8, gestor em saúde)

Não tem contrarreferência. Só com a ginecologista que trabalha no município. (E2, enfermeiro da ESF)

Não, ainda não foi implantado prontuário eletrônico. Existe sim proposta do governo, ainda está muito em discussão, mas na prática isso ainda não aconteceu. (E8, gestor em Saúde)

Além disso, a ausência de prontuários eletrônicos nas USF e a precariedade no compartilhamento das informações clínicas com os demais serviços da rede apresentavam-se como barreiras ao fluxo assistencial. Neste sentido, havia, pontualmente, o recebimento da contrarreferência por meio das tradicionais guias em papel, na dependência da 'boa vontade de alguns profissionais. Portanto, indaga-se sobre as possibilidades de continuidade do cuidado e resolubilidade adequada dos casos numa rede com represamento de informações clínicas.

Na tentativa de superar essa dificuldade, as EqSF buscavam o contato direto com o especialista por meio de telefone pessoal. Caso o contato não fosse possível, aguardavam informações dos usuários trazidas pelo ACS ou agendavam uma visita domiciliar para conversar com o usuário. Esses relatos ampliam as evidências da dificuldade que as EqSF têm para dar continuidade ao cuidado e, sobretudo, garantir uma terapêutica compartilhada entre diferentes especialistas, ratificando achados de outros estudos que apontam para existência de fluxos informais ${ }^{4}$.

Uma medida pró-coordenação é o estabelecimento de acordos entre profissionais da APS e especialistas, com definição de responsabilidades de cada parte ${ }^{8,20}$. Assim, uma melhor coordenação no processo de referência entre médicos da APS e especialistas aumenta a satisfação dos primeiros com a atenção especializada e melhora os resultados obtidos com o encaminhamento ${ }^{20}$. Os autores recém-mencionados apontam, também, que avanços no processo de referência poderiam ser alcançados por meio de melhor comunicação e colaboração entre provedores de diferentes pontos da rede.

O diálogo clínico entre EqSF, núcleo de apoio à saúde da família (NASF) e demais profissionais de outros pontos de atenção deveria ser garantido por mecanismos de comunicação, com o propósito de elaborar 
projetos terapêuticos singulares. Tal comunicação serviria de ajuda para as EqSF, cujas intervenções poderiam alcançar maior resolubilidade, apoiadas em protocolos clínicos com apoio matricial de distintas especialidades. Tratar-se-ia, ainda, de uma medida para diminuir encaminhamentos, tornando-os mais racionais do ponto de vista técnico e econômico, diante da restrita oferta de vagas para as especialidades.

Acerca da existência do diálogo clínico, profissionais manifestaram-se da seguinte forma:

Com as especialidades não [não tem diálogo clínico]. Só com a ginecologista. (E5, enfermeiro da ESF)

[...] Só [existe diálogo clínico] com a ginecologista, porque ela está sempre nas reuniões mensais (E6, enfermeiro da ESF) e [o diálogo clínico acontece] só na reunião mensal. (E4, enfermeiro da ESF)

[O diálogo clinico] é mais com a ginecologista, até porque ela também faz parte do NASF e isso acaba facilitando. (E7, gestor em saúde)

Nota-se que o diálogo clínico entre profissionais de distintos pontos de atenção quando ocorria, ainda era esporádico, tanto por questões relacionadas à própria EqSF quanto em relação aos demais profissionais da rede. Entre os entraves, havia dificuldade de participação de médicos da ESF nas reuniões de equipe, bem como pouca disponibilidade dos profissionais que integram os demais pontos de atenção em interagirem em rede, limitando esse diálogo, muitas vezes, aos trabalhadores envolvidos na APS (ESF e NASF). Por fim, para possibilitar a integração entre profissionais, os sujeitos entrevistados mencionaram iniciativas pontuais, como contatos informais por telefone ou reuniões extraordinárias convocadas pela gestão municipal, mas sem continuidade e sem protocolo/rotina estabelecida nas práticas profissionais.

Em relação ao agendamento das referências, de maneira geral, tanto o aprazamento de consultas das EqSF para a rede especializada local, quanto a marcação de consultas para outros municípios, eram encaminhadas via malote da USF para agendamento na central de regulação e marcação de consultas, uma vez que, nas USF não havia sistema de informação integrado. Entretanto, notou-se que o trabalho das EqSF no agendamento de consultas/procedimentos restringia-se ao envio das solicitações por malote e retorno/ redistribuição aos usuários após autorização, ou seja, sem participação direta das EqSF na definição de prioridades e sem contato com especialistas que pudesse resultar em compartilhamento de informações e condutas terapêuticas.

Outro aspecto importante a ser enfrentado no processo de agendamento de consultas e procedimentos, segundo entrevistados, consistia na solicitação de exames complementares "desnecessários e excessivos. Tal questão, entre outras causas, acentuava-se pela inexistência de protocolos clínicos que dificultava a "padronização técnica de critérios para a solicitação. Além disso, alguns profissionais cediam aos "apelos" dos usuários, como forma de diminuir a pressão cotidiana que a comunidade exerce na prática médica. Um gestor argumenta:

Tem, sempre tem [solicitação de exames desnecessários], mas a gente nunca sabe se realmente aquele paciente vai precisar daquele exame para agora ou para daqui a um mês. Principalmente de exames laboratoriais, porque tem sempre aquele paciente que busca a unidade e quer fazer um check up, aí, o médico pede aquela bateria de exames. (E9, gestor em saúde)

Por sua vez, a regulação do acesso, por meio do processo de agendamento de procedimentos especializados, enfrentava, também, a possibilidade de ausência dos usuários às consultas especializadas. Assim, visando reduzir esse inconveniente e aperfeiçoar a utilização das "escassas" vagas, as EqSF realizavam o monitoramento dos faltosos, para identificação dos motivos que colaboraram para a ausência, bem como, para identificar possível necessidade de reagendamentos, acionando para isso, os ACS. 
Como explicitado nos fragmentos a seguir, as estratégias de monitoramento que as EqSF utilizam estavam mais voltadas para a busca ativa dos usuários. Para tanto, o ACS ia ao encontro do usuário, em sua residência, com a finalidade de obter uma justificativa pelo não comparecimento à consulta agendada, mas, muitas vezes, era o próprio usuário que buscava a USF no intuito de aprazar uma nova data.

É o ACS mesmo que fala se o paciente não foi ou, às vezes, eles [usuários] pegam e trazem [na USF] o papel de novo [...] Ou eles mesmos [usuários] trazem o pedido pra marcar novamente. (E2, enfermeiro da ESF)

Através dos agentes de saúde também. [...] às vezes, quando eles [usuários] faltam eles acabam indo lá com o pedido do exame pedindo a gente para remarcar e justificando por que não foi realizado. (E5, enfermeiro da ESF)

Por fim, os entrevistados afirmaram que, caso o usuário não apresentasse uma justificativa plausível, seria necessário aguardar um intervalo de tempo de três meses para o reagendamento de nova consulta com especialista. Tal questão, derivada de um conjunto de problemas, não resolve a oferta de vagas, "pune o usuário faltoso e, concomitantemente, pode, inclusive, agravar seu problema de saúde, retroalimentando a espiral de problemas na rede.

De acordo com essa ótica, a regulação no setor de saúde deve compreender um arcabouço de atividades, instrumentos e estratégias que visem ao estabelecimento de padrões de qualidade e eficiência da atenção, definição de protocolos clínicos e fluxos assistenciais, regulação dos prestadores de serviços, bem como definição de contratos e formas de pagamento, avaliação das ofertas de insumos e tecnologias, entre outros, com objetivo de garantir acesso integral da população ${ }^{21}$. Tais ações, portanto, têm uma dimensão políticoinstitucional, realizada por gestores dos serviços, e uma dimensão assistencial, exercida no cotidiano dos profissionais.

No município pesquisado, contudo, essas atribuições ficavam sob responsabilidade de profissionais na central de regulação e marcação de consultas, que juntamente com as demais instâncias de gestão gerência da atenção básica à saúde e outras gerências na Secretaria Municipal de Saúde - buscavam monitorar, avaliar e manter atualizados os dados cadastrais do sistema, com base nos relatórios gerados pelos diferentes pontos de atenção.

Nesse sentido, a dimensão da coordenação da área clínica, que exige a participação direta dos profissionais envolvidos no cuidado, resumia a burocracia assistencial:

[O agendamento de consultas é] através da central de marcação. Todos aqueles pedidos que foram feitos durante a semana a gente deixa no malote na unidade. (E5, enfermeiro da ESF)

[...] toda semana, a gente manda esse malote para a central de regulação. A central de regulação faz o agendamento, devolve para a gente com data, horário, local. [...] e toda semana, o ACS vem na unidade, pega e entrega na casa do usuário a confirmação [da consulta]. (E1, enfermeiro da ESF)

Em síntese, as barreiras impostas ao acesso oportuno à atenção especializada são agravadas pela ausência de tecnologias de informação e comunicação (TIC). Por sua vez, no país, resultados do PMAQ-AB evidenciaram que instrumentos elementares como o telefone são pouco utilizados, além de outras formas para marcação de consultas online ${ }^{10}$. Em contrapartida, no cotidiano dos serviços, observa-se que o ACS se tornou o principal intermediário para marcação de consultas, tanto na USF quanto para comunicação do agendamento de procedimentos especializados no território, o que, contraditoriamente, pode comprometer a disponibilidade desse sujeito para o cuidado vigilante no domicílio e gerar uma expectativa 'distorcida no imaginário social em relação ao seu trabalho na ESF. 


\section{Conclusão}

A coordenação do cuidado é um atributo essencial de uma APS forte e, por isso, identificá-la nas práticas das assistências dos profissionais da EqSF sinaliza a capacidade de resolubilidade e continuidade das ações cotidianas.

Com relação a esse aspecto, constatou-se a existência de: ações gerenciais capazes de incidir positivamente sobre a coordenação do cuidado a ser realizada pelas EqSF, a exemplo do uso de orientação institucional para que os usuários busquem as USF como porta de entrada preferencial; central de regulação e marcação de consultas estruturada mediando as relações entre níveis assistenciais; parceria assistencial entre médico e enfermeiro das EqSF nas ações individuais e coletivas; trabalho do ACS relativo à informação comunitária, seja na articulação dos agendamentos na USF, seja na entrega domiciliar de guias de marcação (autorizadas pela regulação) para unidades de outros níveis assistenciais. Porém ainda persistem: um modelo de produção do cuidado por um procedimento-centrado, materializado pelo frágil diálogo clínico entre os profissionais de distintos pontos de atenção; dificuldades na participação de médicos nas reuniões de equipe; dificuldades nas contrarreferências clínicas entre os níveis de atenção e o uso de medidas pontuais para comunicação entre os profissionais.

Cabe reiterar que esses resultados, devidamente contextualizados, podem colaborar tanto para o debate sobre a qualificação dos profissionais da ESF como coordenadores do cuidado clínico, quanto para o aperfeiçoamento do sistema de saúde na perspectiva das redes de atenção integradas, visando ao cuidado continuado dos usuários. Todavia, os resultados apontam aspectos centrados nos instrumentos gerenciais (formulários, fichas, protocolos etc.), em detrimento da gestão clínica no processo de trabalho interprofissional.

Analisar o processo de coordenação de cuidados em cenários reais é necessário, pois as reformas centradas na coordenação dos cuidados também em outros países são incipientes, sobretudo, na América Latina, repercutindo no número reduzido de estudos acerca do assunto. Outrossim, é importante conhecer experiências voltadas para a coordenação do cuidado em saúde, pois muitos desses exemplos podem ser adequados a diferentes realidades, ou até mesmo servir como inspiração para a elaboração de políticas públicas que melhorem o acesso aos níveis de atenção.

De maneira geral, a pesquisa apresentada aqui permitiu constatar a existência de desafios no processo de coordenação do cuidado nas práticas assistenciais. Como principais dificuldades apresentadas, foram mencionados: o modelo biomédico, materializado na predileção pela medicalização e consumo de consultas e procedimentos, presentes na prática profissional e, também, no imaginário cultural dos usuários; as dificuldades na comunicação clínica entre os níveis de atenção, representadas pela ausência de recursos informacionais adequados; o diálogo clínico incipiente entre os profissionais de distintos pontos de atenção, acentuado pela fragmentação da rede e restrição de cotas para acesso aos serviços de maior densidade tecnológica; o uso de medidas pontuais e voluntaristas para comunicação entre os profissionais (telefonemas pessoais, reuniões extraordinárias), sem uma rotina estabelecida no processo de trabalho.

Tais problemas são fragilidades de uma rede municipal que convive com a inexistência de prontuário eletrônico, dificultando o cruzamento de informações compartilhadas pela equipe multiprofissional; com a marcação de consultas para cuidados especializados restrita à central de marcação, não havendo participação direta das EqSF na definição de prioridades; com a inexistência de telessaúde; e com a resistência ao SUS por parte de alguns usuários que possuem seguro privado de saúde.

Nessa perspectiva, há evidências contundentes da fragilidade da APS em pequenos municípios, mesmo que tenham cobertura plena dos seus cidadãos em relação à ESF. Grosso modo, a ausência de retaguarda assistencial e o acesso desarticulado à atenção secundária não viabilizam a coordenação, nem tampouco permitem o cuidado longitudinal. Desse modo, amontoam-se milhares de microssistemas de baixa 
resolubilidade, numa espiral de problemas que desagrada profissionais e usuários, fortalecendo o discurso contra o sistema público de saúde.

Por essa ótica, gestores de pequenos municípios precisam de sistemas colaborativos entre si e entre municípios de maior porte, por meio de territórios sanitários regionalizados, para viabilidade técnica e tecnológica dos diferentes meios para produção do cuidado. Ainda assim, a existência de profissionais e equipamentos de diferentes densidades tecnológicas não será capaz de garantir a resolubilidade se estiverem fragmentados e sem integração assistencial. Em síntese, necessita-se de uma APS fortemente articulada com os diferentes níveis de atenção que, por sua vez, carecem de coordenação gerencial (autoridade sanitária) e coordenação clínica (interprofissionais) para evitar sobreposição terapêutica, garantir o acesso oportuno e, por fim, resolver os problemas de saúde da população.

\section{Referências}

1. Spedo SM, Pinto NRS, Tanaka OY. O difícil acesso a serviços de média complexidade no SUS: o caso da cidade de São Paulo, Brasil. Physis. 2010;20(3):953-72.

2. Mendes EV. As redes de atenção à saúde. Ci Saúde Coletiva. 2010;15(5):2297-05.

3. Nova Comissão Nacional de Incorporação de Tecnologias de Saúde e Impacto ao Sistema Único de Saúde. R Saúde Pública. 2011;45(5):993-6.

4. Santos AM, Giovanella L. Governança regional: estratégias e disputas para gestão em saúde. R Saúde Pública. 2014;48(4):622-31.

5. Almeida PF, Giovanella L, Mendonça MHM, Escorel S. Desafios à coordenação dos cuidados em saúde: estratégias de integração entre níveis assistenciais em grandes centros urbanos. Cad Saúde Pública.2010 fev.;26(2):286-98.

6. Gress $\mathrm{S}$, Baan CA, Calnan M, Dedeu T, Groenwegen P, Howson $\mathrm{H}$ et al. Co-ordination and management of chronic conditions in Europe: the role of primary care - position paper of the European Forum for Primary Care. Qual Prim Care. 2009;17(1):75-86.

7. Kringos DS. The strength of primary care in Europe. In: Kringos DS, Boerma WGW, van der Zee J, Groenewegen PP. The contribution of primary care to health care system performance in Europe. Utrecht: Nivel; 2012. p. 217-38.

8. Bodenheimer T. Coordinating care - a perilous journey through the health care system. N Engl J Med. 2008 Mar;358(10):1064-71.

9. Starfield B. Atenção primária: equilíbrio entre as necessidades de saúde, serviços e tecnologia. Brasília: Unesco, Ministério da Saúde; 2002.

10. Fausto MCR, Giovanella L, Mendonça MHM, Seid H, Gagno J.A posição da estratégia saúde da família na rede de atenção à saúde na perspectiva das equipes e usuários participantes do PMAQ-AB. Saúde Debate. 2014;38(n. especial):13-33.

11. Franco TB. Transição tecnológica e inclusão social na saúde. In: Franco TB, Andrade CS, Ferreira VSC(Orgs.). A produção subjetiva do cuidado: cartografias da Estratégia Saúde da Família. São Paulo: Hucitec; 2009.

12. Almeida $\mathrm{PF}$, Santos $\mathrm{AM}$, Souza $\mathrm{MKB}$, organizadores. Atenção primária à saúde na coordenação do cuidado em regiões de saúde. Salvador: Edufba; 2015.

13. Minayo MCS. O desafio do conhecimento: pesquisa qualitativa em saúde. 10. ed. São Paulo: Hucitec; 2007.

14. Thiry-Cherques HR. Saturação em pesquisa qualitativa: estimativa empírica de dimensionamento. AfRevista PMKT. 2009;3:20-7.

15. Arce VAR, Sousa MF. Práticas de longitudinalidade no âmbito da estratégia saúde da família no Distrito Federal. Cad Saúde Colet. 2014;22(1):62-8.

16. Cecílio LC. E. A morte de Ivan Ilitch, de Leon Tolstói: elementos para se pensar as múltiplas dimensões da gestão do cuidado. Interface. 2009;13(suppl.1):545-55. 
17. Santos AM, Kochergin CN, Jesus SR, Soares DA, Assis MMA, Bispo Júnior JP et al. Linhas do cuidado e responsabilização no PSF de um município da região Sudoeste da Bahia: um olhar analisador. In: Assis MMA, Nascimento MAA, Franco TB, Jorge MSB, organizadores. Produção do cuidado no programa saúde da família: olhares analisadores em diferentes cenários. Salvador: Edufba; 2010. p. 59-82.

18. Giovanella L. Atenção primária à saúde e coordenação dos cuidados na rede assistencial. Divulg. Saúde Debate. 2014;(51):30-7.

19. Costa SL, Carvalho EN. Agentes comunitários de saúde: agenciadores de encontros entre territórios. C Saúde Coletiva.2012;17(11):2931-40.

20. Forrest CB, Glade GB, Baker AE, Bocian A, von Schrader S, Starfield B. Coordination of specialty referrals and physician satisfaction with referral care. Arch Pediatr Adolesc Med. 2000;154(5):499-06.

21. Conselho Nacional de Secretários de Saúde. Regulação em Saúde. Brasília; 2011. (Coleção Para entender a gestão do SUS 2011; 10). 